\title{
Three dimensional analysis of flow around a porous spur dike
}

\author{
Tomasz MIODUSZEWSKI ${ }^{1}$ and Shiro MAENO ${ }^{2}$
}

\author{
${ }^{1}$ Dr. of Eng., e-mail: tomasz@aquaprojekt.pl \\ AQUAPROJEKT Ltd., Hydro-Engineering Design Office, ul. Slupska 72, 80-392 Gdansk, Poland \\ ${ }^{2}$ Member of JSCE, Dr. of Eng., Associate Professor, e-mail: maeno@cc.okayama-u.ac.jp \\ Dept. of Civil Engineering, Okayama University, Tsushima Naka 3-1-1, Okayama 700-8530, Japan
}

\begin{abstract}
The goal of study described in this paper is to develop the three dimensional analytical model to simulate a flow around a porous structures. The VOF method was used for the free surface flow field considering the future applicability to the rapid changes of the flow such as a surge flow. The flow in porous media was modeled by the Brinkman-Forchheimer extended Darcy's equation. As the experimental verification, three dimensional velocities around a spur dike were measured by using Acoustic-Doppler Velocimeter (ADV). The numerically obtained flow profile around a permeable spur dike was compared with the experimental results. It is confirmed that agreement between proposed numerical result and experiment is good under sub-critical flow condition. Some limitations according to mesh size and boundaries were exposed, nevertheless applicability of the model was proved. The present study enables the future simulation of rapidly varied flow around permeable hydraulic structures.
\end{abstract}

Key Words: porous spur dike, three dimensional flow, numerical analysis, $V O F$, porous hydraulic structure

\section{Introduction}

Spur dikes are seen as the best type of the hydro-engineering river training structures that allow redirecting the flow of water in a river or in a channel. Deflecting of the current is very important and advisable in many cases. The best example is when the flow of the stream should be directed away from the bank to protect it from erosion. The usage of a set of several spur dikes is one of the most effective means of stabilizing the river or channel banks.

In recent years the researchers focused on study of possible advantages of environment-friendly hydraulic structures made of natural material such as a rubble mound weirs. Also the researchers in Netherlands pointed a need of innovations in spur design. Several methods of the groyne design innovation were presented by Van der $\mathrm{Wal}^{1)}$. One of the most important remarks was to make the structure permeable. The same suggestion was seen by the American researchers, resulting in the description of the spur's permeability in Hydraulic Engineering Circular No. 23 by Lagasse et al. ${ }^{2)}$. In the previous research projects, the authors ${ }^{3), 4)}$ also pointed the advantage of the structure's permeability resulting in reduction of the scouring around the spur dike. Also the difference in flow pattern during rapid flow cases (the most dangerous for the structure's stability) was recognized.

Considering the above mentioned background, development of a three dimensional numerical model to analyze the flow around permeable structures becomes an important and needed task.

\section{Numerical and Experimental Procedure}

\subsection{Governing equations for fluid phase ${ }^{5), 6), 7)}$}

Basic equations used in the calculation are momentum, continuity and transport equation of $F$ quantity ( $F$ is fractional volume of fluid). Standard $k-\varepsilon$ model is used as a turbulence model.

Continuity equation:

$$
\frac{\partial u_{j}}{\partial x_{j}}=0
$$

Momentum equations:

$$
\begin{gathered}
\frac{\partial u_{i}}{\partial t}+u_{j} \frac{\partial u_{i}}{\partial x_{j}}=F_{i}-\frac{1}{\rho} \frac{\partial P}{\partial x_{i}}+ \\
\left(v+v_{t}\right) \frac{\partial}{\partial x_{j}}\left(\frac{\partial u_{j}}{\partial x_{i}}+\frac{\partial u_{i}}{\partial x_{j}}\right)-\frac{2}{3} \frac{\partial k}{\partial x_{j}}
\end{gathered}
$$

Transport equation of $F$ :

$$
\frac{\partial F}{\partial t}+\frac{\partial u_{j} F}{\partial x_{j}}=0
$$


$k$-equation:

$$
\frac{\partial k}{\partial t}+u_{j} \frac{\partial k}{\partial x}=\frac{\partial}{\partial x_{j}}\left[v_{k} \frac{\partial k}{\partial x_{j}}\right]+v_{t} \frac{\partial u_{i}}{\partial x_{j}}\left(\frac{\partial u_{i}}{\partial x_{j}}+\frac{\partial u_{j}}{\partial x_{i}}\right)-\varepsilon
$$

$\varepsilon$-equation :

$$
\begin{gathered}
\frac{\partial \varepsilon}{\partial t}+u_{j} \frac{\partial \varepsilon}{\partial x}=\frac{\partial}{\partial x_{j}}\left[v_{\varepsilon} \frac{\partial \varepsilon}{\partial x_{j}}\right]+ \\
C_{1} \frac{\varepsilon}{k} v_{t} \frac{\partial u_{i}}{\partial x_{j}}\left(\frac{\partial u_{i}}{\partial x_{j}}+\frac{\partial u_{j}}{\partial x_{i}}\right)-C_{2} \frac{\varepsilon^{2}}{k} \\
v_{t}=C_{u}\left(k^{2} / \varepsilon\right), \\
v_{k}=v+v_{t} / \sigma_{\kappa}, \quad v_{\varepsilon}=v+v_{t} / \sigma_{\varepsilon}
\end{gathered}
$$

where, $i, j$ : Cartesian tensor indices, $x_{j}$ : Cartesian coordinate, $u_{j}$ : mean velocities, $\rho$ : density of fluid, $P=p+2 / 3 \cdot \rho k, p$ : fluid pressure, $g$ : acceleration due to gravity, $F_{i}$ : body force components, $k$ : kinetic energy, $\varepsilon$. kinetic energy dissipation rate, $v$. fluid kinematic viscosity, $v_{t}$ : eddy viscosity.

Standard values of constants in the $k$ - $\varepsilon$ model are used: $C_{\mu}=0.09, \sigma_{\kappa}=1.00, \sigma_{\varepsilon}=1.30, C_{l}=1.44, C_{2}=1.92$

\subsection{Governing equations for porous phase}

Effective pore fluid velocity $u_{i}$ is given by volume-averaged seepage velocity $u_{s i}$ and porosity of the porous medium $n$.

$$
u_{i}=u_{s i} / n
$$

Using Eq.(7) following equations are obtained.

Continuity equation:

$$
\frac{\partial u_{s j}}{\partial x_{j}}=0
$$

Momentum equations:

$$
\begin{gathered}
n \frac{\partial u_{s i}}{\partial t}+u_{s j} \frac{\partial u_{s i}}{\partial x_{j}}=n F_{i}-\frac{n^{2}}{\rho} \frac{\partial p}{\partial x_{i}}+ \\
n\left(v+v_{t}\right) \frac{\partial}{\partial x_{j}}\left(\frac{\partial u_{s j}}{\partial x_{i}}+\frac{\partial u_{s i}}{\partial x_{j}}\right)- \\
n^{2}\left(\frac{v}{\kappa}+\frac{C_{F}}{\sqrt{\kappa}} \sqrt{u_{s j} u_{s j}}\right) u_{s i}
\end{gathered}
$$

Transport equation of $F$ :

$$
\frac{\partial F}{\partial t}+\frac{\partial\left(u_{s j} F\right)}{\partial x_{j}}=0
$$

$k$-equation:

$$
\begin{gathered}
n \frac{\partial k}{\partial t}+u_{s j} \frac{\partial k}{\partial x}=n \frac{\partial}{\partial x_{j}}\left(v_{k} \frac{\partial k}{\partial x_{j}}\right)+ \\
v_{t} \frac{\partial u_{s i}}{\partial x_{j}}\left(\frac{\partial u_{s i}}{\partial x_{j}}+\frac{\partial u_{s j}}{\partial x_{i}}\right)-n \varepsilon-2 n^{2}\left\{\frac{v}{K}+\frac{C_{F}}{\sqrt{K}} \sqrt{u_{s j} u_{s j}}\right\} k
\end{gathered}
$$

$\varepsilon$-equation:

$$
\begin{gathered}
n \frac{\partial \varepsilon}{\partial t}+u_{s j} \frac{\partial \varepsilon}{\partial x}=n \frac{\partial}{\partial x_{j}}\left(v_{\varepsilon} \frac{\partial \varepsilon}{\partial x_{j}}\right)+ \\
C_{1} \frac{\varepsilon}{k} v_{t} \frac{\partial u_{s i}}{\partial x_{j}}\left(\frac{\partial u_{s i}}{\partial x_{j}}+\frac{\partial u_{s j}}{\partial x_{i}}\right)-n C_{2} \frac{\varepsilon^{2}}{k}- \\
2 n^{2}\left\{\frac{v}{K}+\frac{C_{F}}{\sqrt{K}} \sqrt{u_{s j} u_{s j}}\right\} \varepsilon
\end{gathered}
$$

Last term of Eqs. (9), (11) and (12) is Brinkman-Forchheimer extended Darcy's resistance form. It is composed of the resistance components of both: laminar and turbulent flow through the porous medium. $K$ is a permeability parameter with the dimension of square of length. It is given by the following expression as a function of mean sand diameter $d_{m}$ :

$$
\sqrt{\kappa}=e \cdot d_{m}
$$

where $e$ was identified through the authors' experiment on rubble mound weir ${ }^{8)}$ to be: $e=0.015$.

Coefficient $C_{F}$ is the Forheimer number and as suggested by E.C. Chan ${ }^{9)}$ was set to $C_{F}=0.1$. Finite difference method was used in the calculation, and second order centered difference scheme was used for the convection term.

\subsection{Experimental setup}

The flume is a $15 \mathrm{~m}$ long, $0.6 \mathrm{~m}$ wide and $0.4 \mathrm{~m}$ deep steel construction with its front wall made of glass. The channel inclination was $1 / 500$. The pit for experimental installation is located in the middle part of the flume. The depth of the pit is $17 \mathrm{~cm}$ and its length is $100 \mathrm{~cm}$ (see Fig. 1). Its width is equal to the channel width. During the experiment the pit was filled with movable sand. The sand used for this experiment is characterized by an averagc grain diameter $d_{50}=1.28 \mathrm{~mm}$. To focus on the velocity field the sand was covered with steel mesh preventing scouring process. The mesh used in the experiment was very thin, therefore, its effect considered to be very small. Its porosity allowed water to flow to and from the sandy bed below. Roughness of such a surface was different from the roughness of sand, but as the investigation of the roughness was not in a scope of this research, the effect was not taken under consideration.

The spur dike was constructed as a full height structure, $6 \mathrm{~cm}$ thick and $15 \mathrm{~cm}$ wide. Two types of the spur dikes were used in this experiment (Fig. 1). The impermeable spur dike was solid structure made of Plexiglas. The permeable one was made of steel mesh filled with stones (average size $2 \mathrm{~cm}$ ).

The measurement area of velocity field is presented in Fig. 2. The measurement started $1.0 \mathrm{~m}$ upstream from the dike and last points were located 


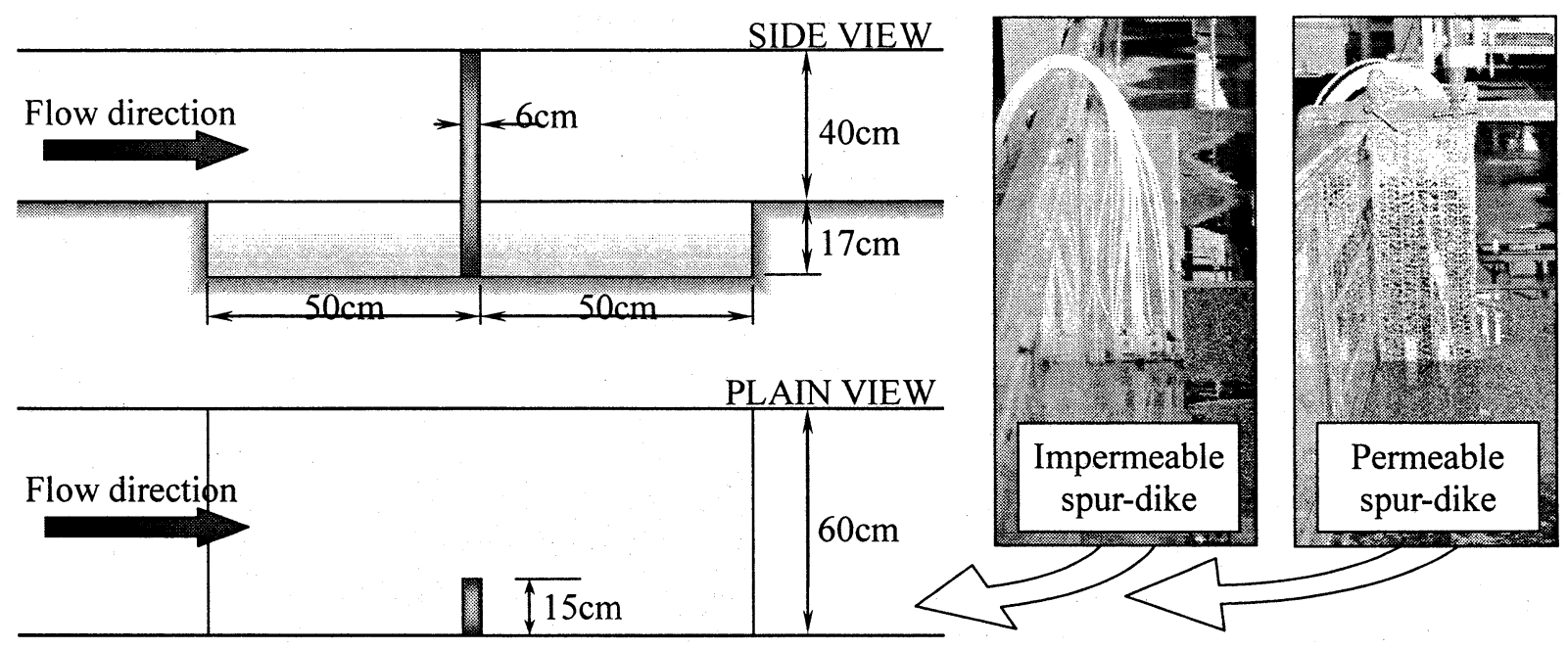

Fig. 1 Experimental setup
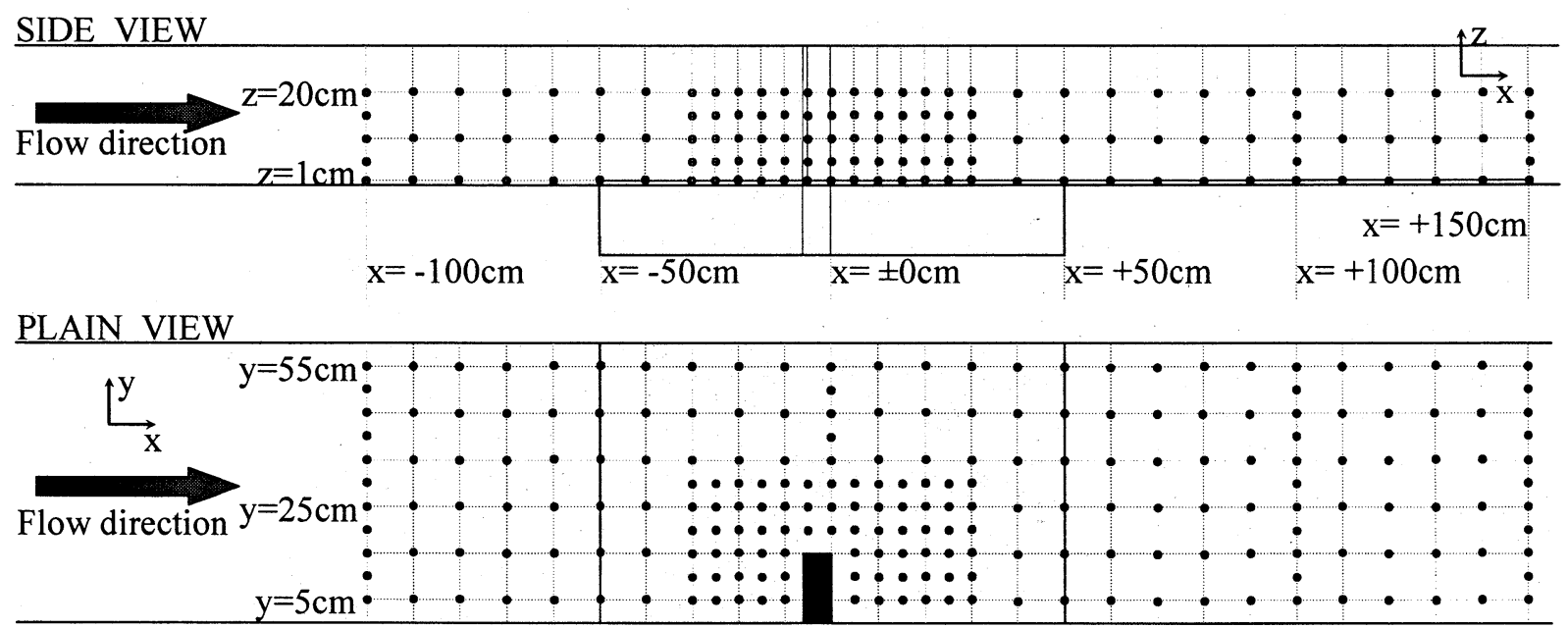

Fig. 2 Location of velocity measurement points

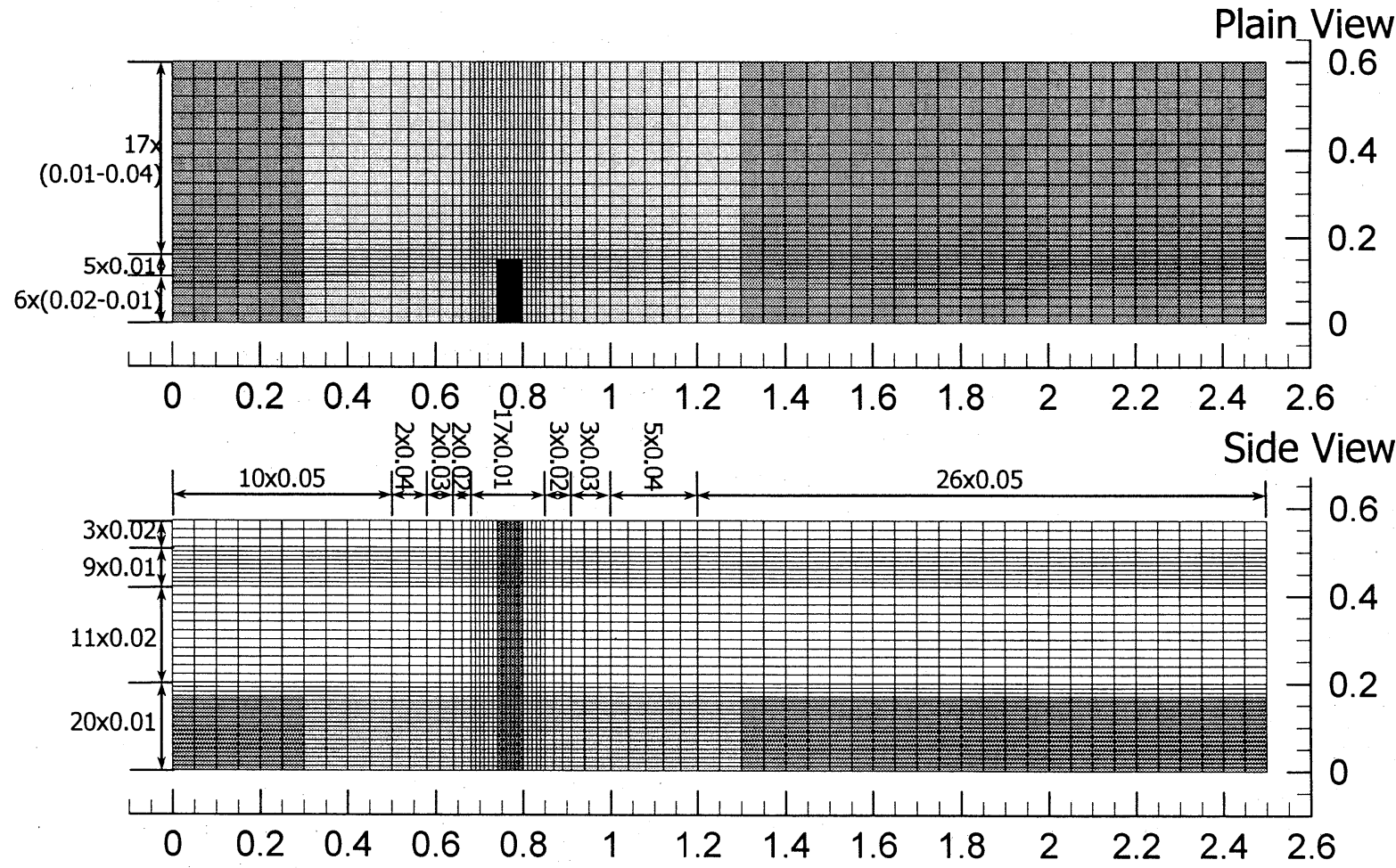

Fig. 3 Calculation mesh (unit: m) 
$1.5 \mathrm{~m}$ downstream form the dike. Basic grid of measurements was set to $0.1 \times 0.1 \times 0.1 \mathrm{~m}$. The area in the close vicinity to the spur (from $0.3 \mathrm{~m}$ upstream to $0.3 \mathrm{~m}$ downstream, and $50 \%$ of the channel width) was covered by the measurement in the grid $0.05 \times 0.05 \times 0.05 \mathrm{~m}$. Also the initial upstream $(x=-1.0 \mathrm{~m})$, center $(x= \pm 0.0 \mathrm{~m})$, and two downstream cross-sections $(x=+1.0 \mathrm{~m} \mathrm{\&} x=+1.5 \mathrm{~m})$ were covered by the grid $0.05 \times 0.05 \mathrm{~m}$. The velocity was measured using Acoustic Doppler Velocimeter (ADV).

Stable discharge was set to $Q=0.02 \mathrm{~m}^{3} / \mathrm{s}$ during all the measurement. Depth was $0.3 \mathrm{~m}$ at $x=-1.0 \mathrm{~m}$. The depth condition was controlled by the tail gate at the end of experimental flume, $x=5.1 \mathrm{~m}$.

\subsection{Numerical conditions}

Considering the computer memory and time consumption, the region of calculation was limited to the area shown in Fig. 3. As shown in the figure, variable mesh was used, that is the mesh size becomes smaller around the spur dike. In total, the area of calculation was composed of 84280 cells (including 13440 obstacle cells). Uniform velocity $V_{\text {ini }}=0.04 \mathrm{~m} / \mathrm{s}$ was applied to fluid phase as an initial condition in all the area. Uniform velocity $V_{\text {in }}=0.11 \mathrm{~m} / \mathrm{s}$ was applied at the inflow boundary. At the outflow boundary, considering the limited distance from the spur dike, the logarithmic velocity profile was set with the constant outflow rate $Q=0.02 \mathrm{~m}^{3} / \mathrm{s}$.

After testing many combinations, logarithmic condition was applied to the spur-dike walls and fixed bed. At the sidewalls of the flume the slip condition was set. Time step was constant and was equal to $2.0 \times 10^{-4} \mathrm{~s}$.

The sand used in both cases as the riverbed in the vicinity of the structure was characterized by porosity 0.4 and permeability $K=3.7 \times 10^{-10} \mathrm{~m}^{2}$. In the case of permeable spur, the model was described by porosity 0.4 and permeability $K=7.3 \times 10^{-8} \mathrm{~m}^{2}$.

\section{Results and Discussion}

Fig.4 shows the discharge variation with time at several cross sections in the impermeable spur dike case. Large fluctuation of the discharge can be seen in the early stage of calculation. After that, discharge fluctuation disappears and converges to the inflow and outflow discharge given as a boundary condition. The discharge fluctuation shows that the calculation is stabilized after $10 \mathrm{~s}$. Although it is not shown in this paper, the discharge fluctuation pattern with permeable spur dike is very similar to the presented one.

The comparison between measured (thick line) and computed (thin line) velocities for the impermeable structure's case is presented in Fig. 5 and Fig. 6. Velocity vectors in the following figures are shown in the same manner. Fig. 5 shows the results at fully stabilized step of calculations $(t=15 \mathrm{~s})$, and Fig. 6 shows at the end of stabilization phase $(t=8 \mathrm{~s})$. In the author's opinion, the state not fully stabilized reproduces experimental findings in much better way, as some fluctuation also were noticed during the experiment. In the results after full stabilization the flux in the area just behind the dike returns to the channel wall. The vortex is very small and concentrated close to the downstream-sidewall of the dike. The main reason was described by $\mathrm{Hirt}^{10)}$ as an effect of truncation errors called numerical dispersion or numerical smoothing. As stated before, because of very small mesh and three-dimensional domain the time step have had very small value. As a result, the flux just after the obstacle is not forming an continuous path of similar width, but is dispersing very quickly. This dispersion was not so strong during the stabilization phase.

Another possible reason for the return of the flux is the boundary condition at the spur's headwall. The logarithmic boundary condition was used. In general, boundary condition at the wall forces the fluid to move only in direction normal to the wall. According to this rule, at the headwall of the dike, water movement is limited to the $\mathrm{x}$ and $\mathrm{z}$ direction. In other words the layer of the thickness of $1 \mathrm{~cm}$ (size of the cell in y direction) is the boundary layer. The boundary layer in the experimental condition is assumed to be much thinner, especially at the smooth acrylic surface which was investigated in the experiment. Then, amount of momentum is very different, as the amount of water in a boundary layer is different.

Those two effects combined together gave a result of huge numerical smoothing. That is why, the results obtained at the time $t=15 \mathrm{~s}$ are over-smoothed. In the case of permeable structure, as the whole area (including the structure) is treated as a fluid there was no boundary layer at the headwall of the dike. Therefore, the results at $t=15 \mathrm{~s}$ were not affected by the effect of numerical problems. In the graphs showing the permeable case results, the time $t=15 \mathrm{~s}$ is presented.

The velocity magnitudes from the experiment and simulation are very similar. For the impermeable structure case the velocity maps showing magnitudes of velocities $V_{x}, V_{y}$ and $V_{z}$ are presented in Figs. 7, 8 and 9 respectively. Some discontinuities of the lines in the experimental results are an effect of different grid size in different areas and overlapping of those areas. Following the assumption stated above that the calculation at the 


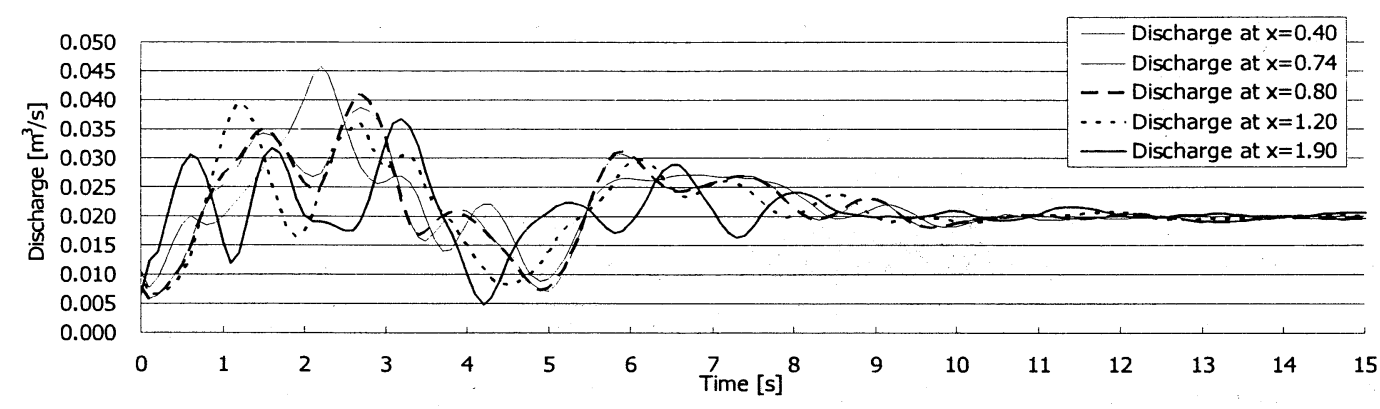

Fig. 4 Discharge fluctuation in the early stage of calculation

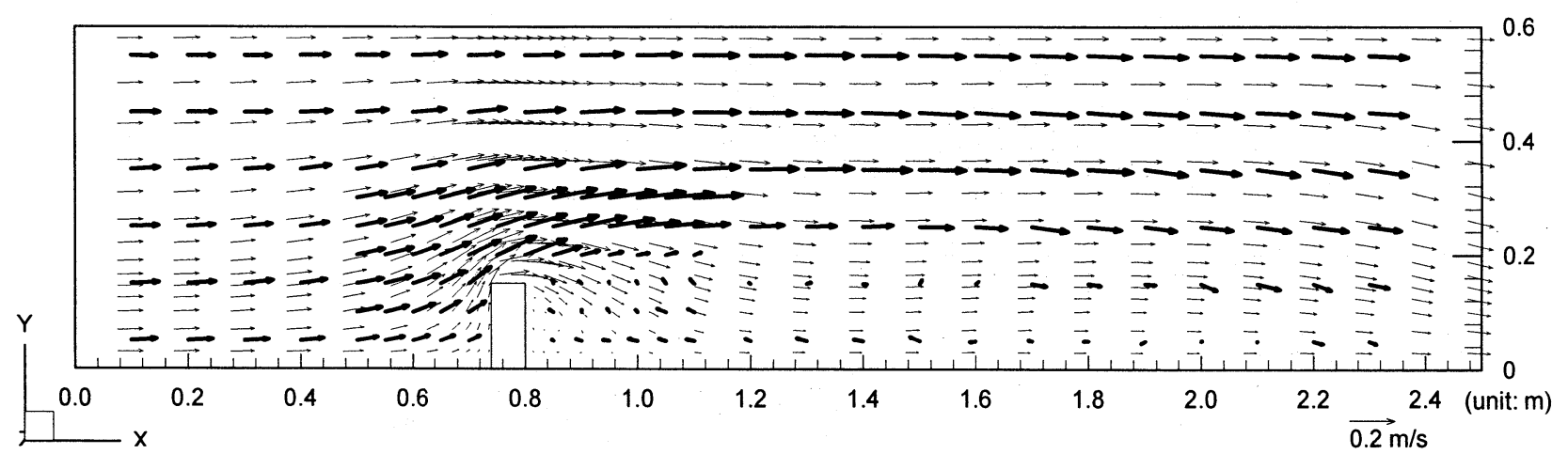

Fig. 5 Simulated ( $t=15 \mathrm{~s}$ ) and measured velocity field; plain view at $z=0.1 \mathrm{~m}$ above the bed; impermeable spur dike

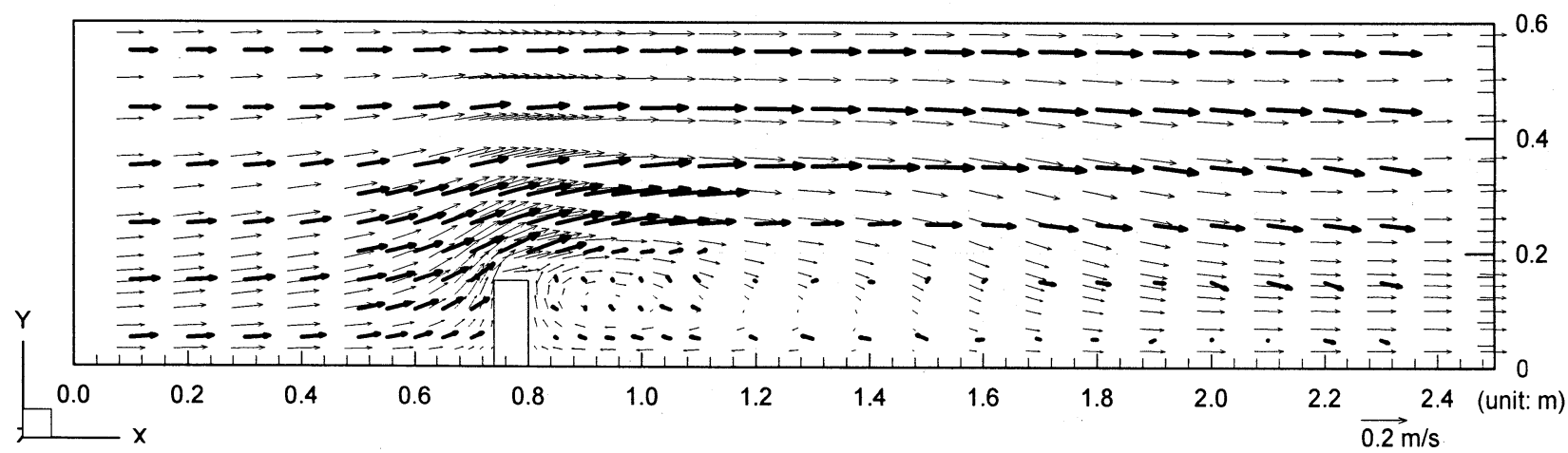

Fig. 6 Simulated $(t=8 \mathrm{~s}$ ) and measured velocity field; plain view at $\mathrm{z}=0.1 \mathrm{~m}$ above the bed; impermeable spur dike

end of stabilization phase $(t=8 \mathrm{~s})$ reproduces experimental results better than fully stabilized state was confirmed and only this stage is presented in the figures. The overall pattern of the velocity magnitudes is reproduced in satisfactory manner. The high velocity area $\left(V_{x}>0.15 \mathrm{~m} / \mathrm{s}\right)$ located at the side of the channel opposite to the spur dike is visible in the simulation as well as in the experimental results. The influence of the downstream boundary condition is recognized in computational results. Uniform velocity profile at the whole width of the channel's outflow boundary suppresses the high velocity area as well as the vortex area. Limitations of the experimental tools didn't allow the measurement of the velocity very close to the channel's and spur's walls. That is why in Fig. 7a the negative current below $0.05 \mathrm{~m} / \mathrm{s}$ is not visible in this scale. The position of the iso-line representing zero velocity proves good agreement between calculation and experiment.
The detailed analysis of Fig. 8 leads to similar conclusions. The range of the area influenced by the spur is similar near the tip of the dike and at the upstream side of the structure. The downstream side is influenced by the outflow condition. The distance, at which the positive velocities were recorded, is shorter especially in the region, where the flux is the strongest. Also the dark area on the graph representing negative current (the flux returning to the channel's right-hand-side wall) is much closer to the dike. In the experimental results, it was observed around the $x=2.1 \mathrm{~m}$ in the central part of the flume, while in the numerical results $(t=8 \mathrm{~s})$ it was located around $x=1.7 \mathrm{~m}$. It is not shown in this paper, but for the fully stabilized state, the returning current in $y$-direction was noticed just behind the dike.

The strength of the deflection in front of the dike in calculation is also in good agreement with the experimental data. The highest velocities were observed in the very close vicinity of the structure, 

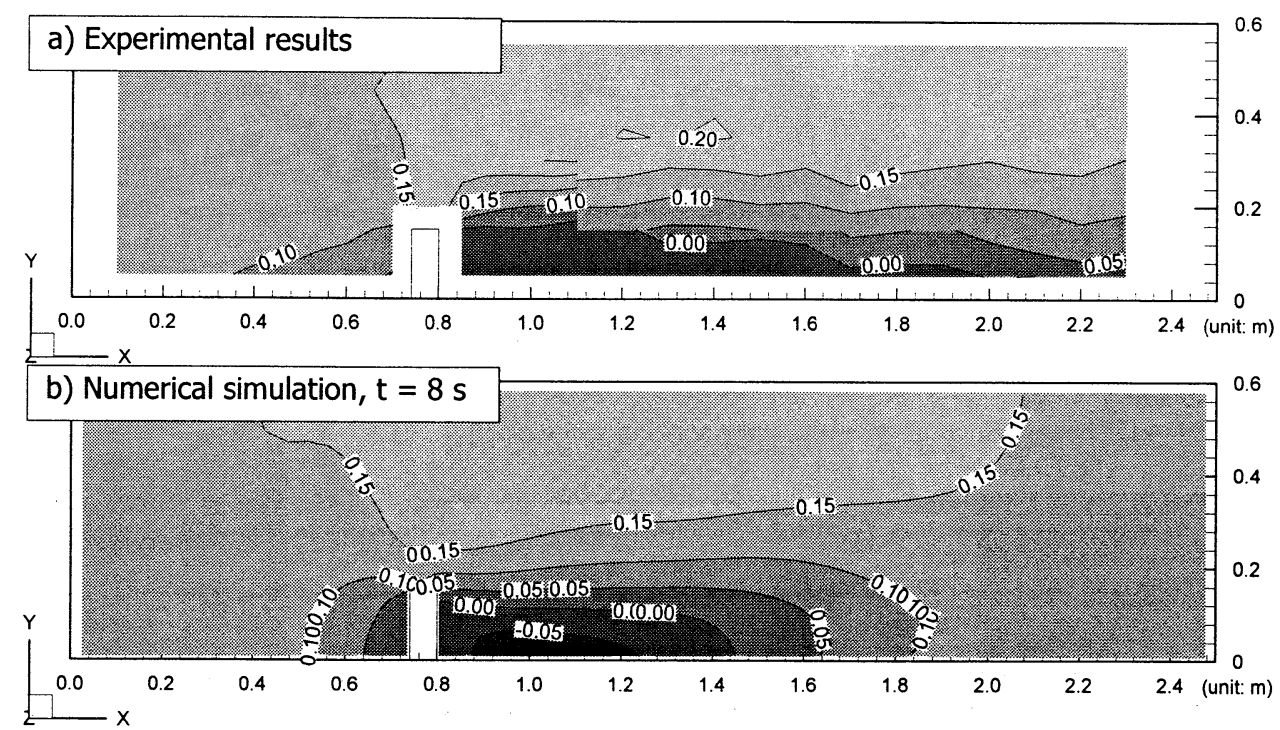

Fig. 7 Magnitude of horizontal, longitudinal velocity $V_{x}$ in case of impermeable structure at $z=0.1 \mathrm{~m}$
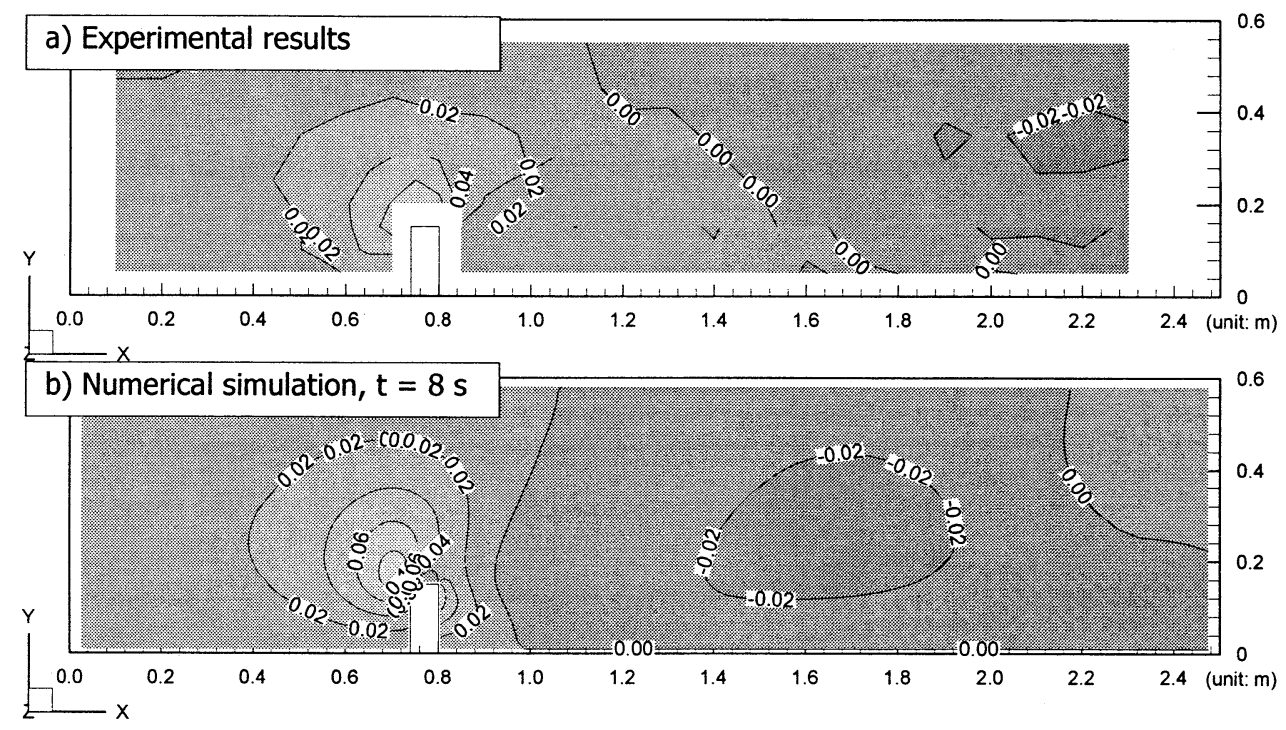

Fig. 8 Magnitude of horizontal, transversal velocity $V y$ in case of impermeable structure at $z=0.1 \mathrm{~m}$

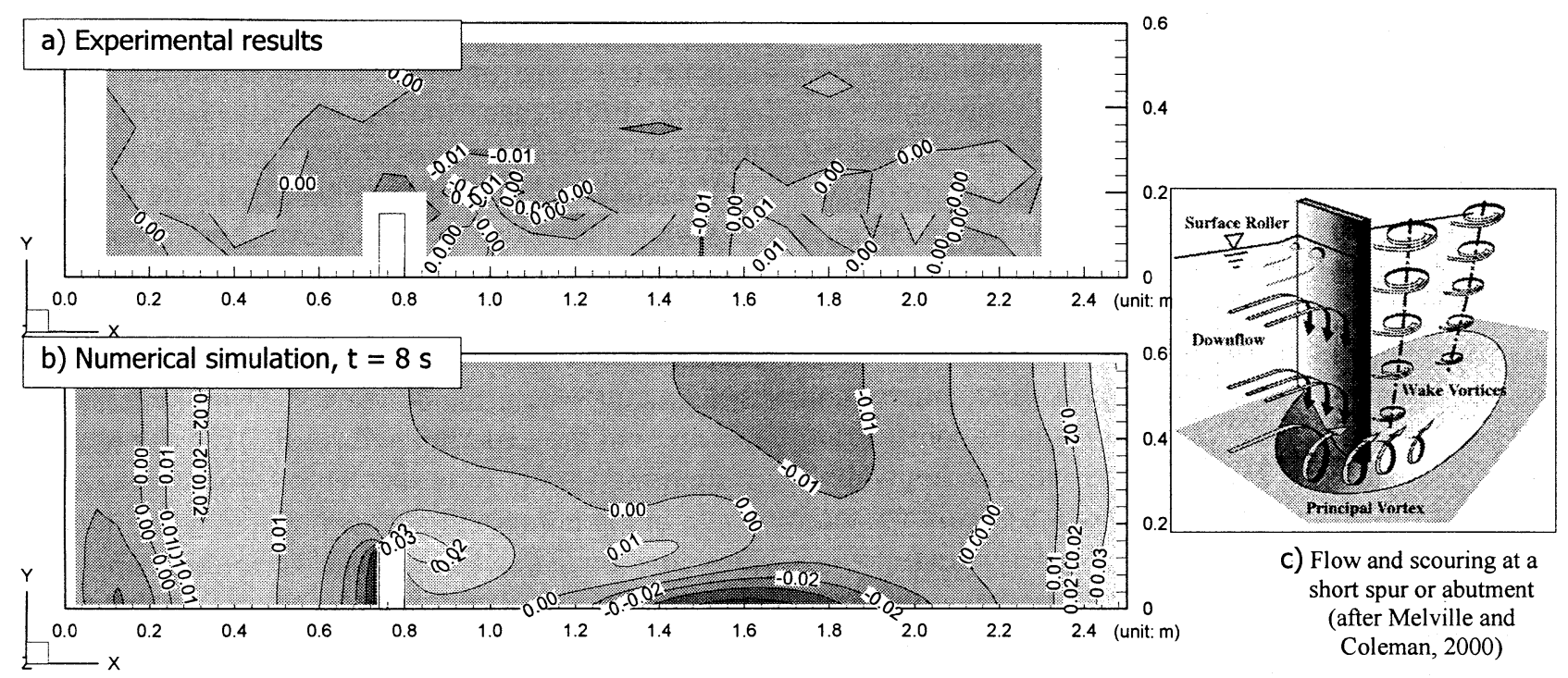

Fig. 9 Magnitude of vertical velocity $V z$ in case of impermeable structure at $z=0.1 \mathrm{~m}$ 


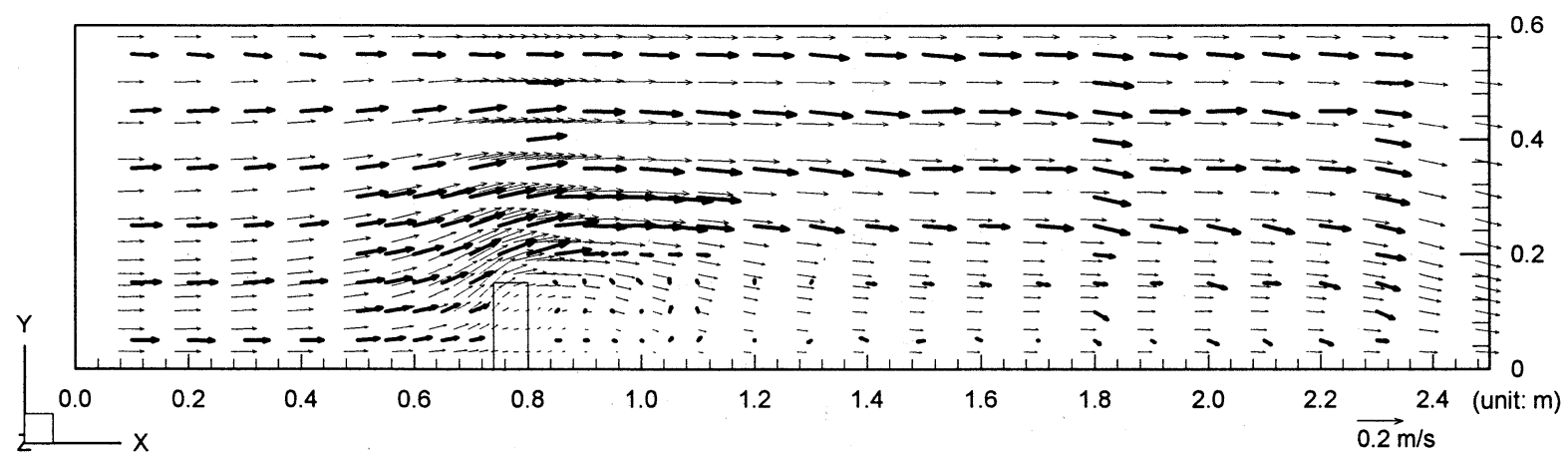

Fig. 10 Simulated $(t=15 \mathrm{~s})$ and measured velocity field; plain view at $\mathrm{z}=0.1 \mathrm{~m}$ above the bed; permeable spur dike

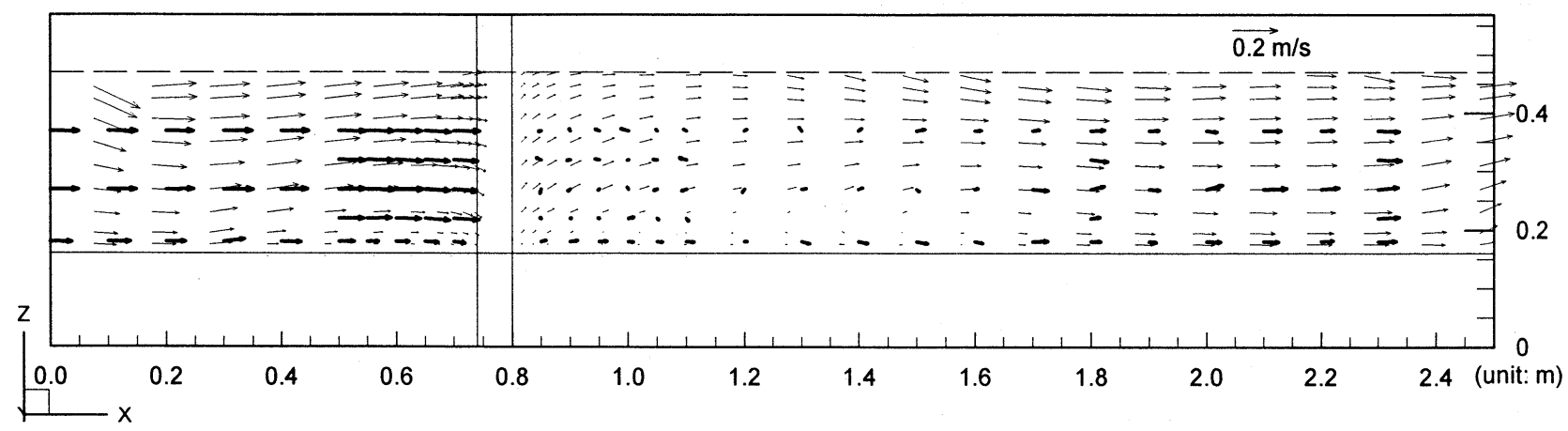

Fig. 11 Simulated $(t=8 \mathrm{~s})$ and measured velocity field - cross-section at $\mathrm{y}=0.15 \mathrm{~m}$ - impermeable spur dike

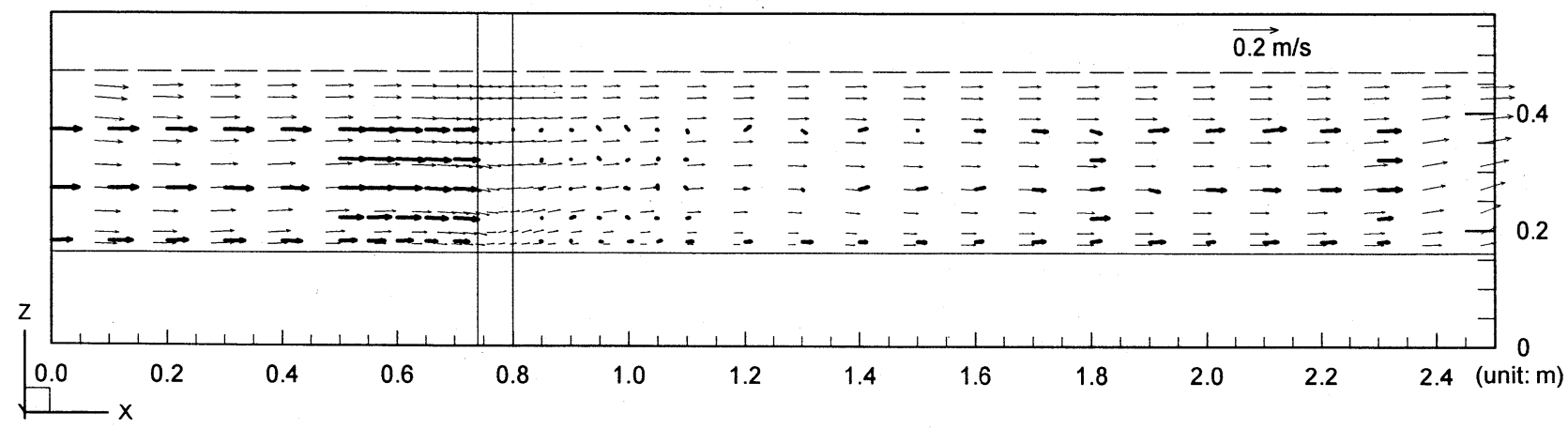

Fig. 12 Simulated $(t=15 \mathrm{~s})$ and measured velocity field - cross-section at $y=0.15 \mathrm{~m}$ - permeable spur dike

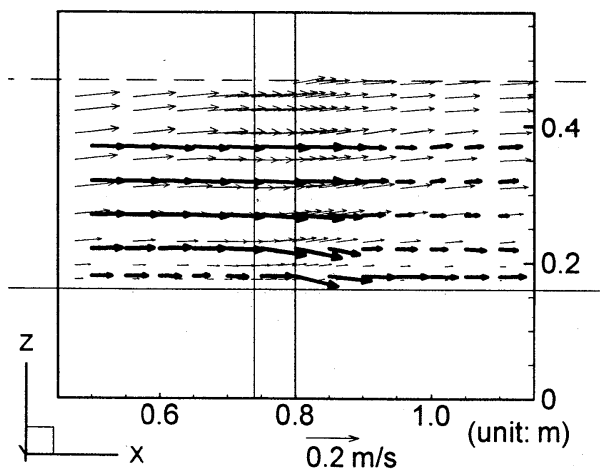

Fig. 13 Simulated and measured velocity field - cross-section at $y=0.20 \mathrm{~m}$ near the spur dike head - impermeable spur dike

at the edge of headwall and upstream-sidewall. The limitations of the experimental tools did not allow measuring the velocity in such a distance, but the patterns formed by the iso-lines of lower velocities confirm reasonably accurateness of calculations.

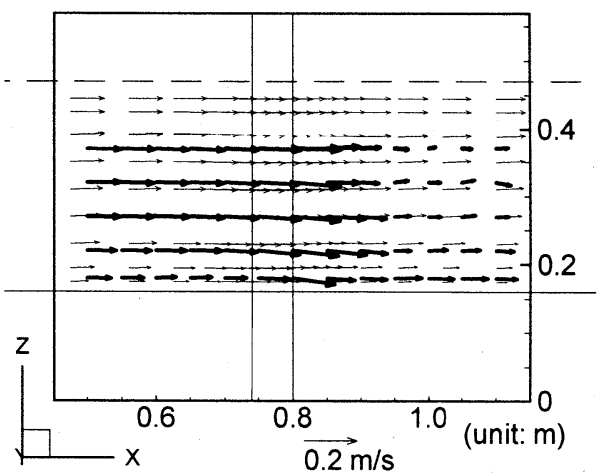

Fig. 14 Simulated and measured velocity field - cross-section at $\mathrm{y}=0.20 \mathrm{~m}$ near the spur dike head - permeable spur dike

The contour plots of the vertical velocity are presented in Fig. 9. Generally speaking, the magnitudes of vertical velocities at the elevation $0.1 \mathrm{~m}$ above the riverbed were very small. In the numerical simulations (see Fig. 9b) the influence of 
the boundary conditions was clearly visible. Near the inflow boundary the flux was directed slightly downward, while at the end of the numerical flume the flux pointed upward. Near the structure, in numerical results, the flow directed downward (up to the value of $V_{z}=-0.04 \mathrm{~m} / \mathrm{s}$ ) at the upstream side and upward at the downstream side. In the experimental data this trend was not seen at the elevation presented in above figures, because the limitation of the measurement tool did not allowed to measure velocities very close to the structure. On the other hand, other researchers noted the trends seen in numerical results. Melville and Colleman ${ }^{11)}$ described the downward current near the upstream sidewall of a short structure (see Fig. 9c). At other levels similar pattern was observed (compare Fig. 11 and Fig. 13).

The difference between permeable and impermeable case was very small in both numerical and experimental results. Nevertheless some general remarks are to be stated. Results of calculation with referential experimental data are presented in Fig. 10. In experimental results the deflection of the current in case of impermeable structure is larger compared with permeable case. Also the range of the vortex is slightly larger. The simulation results followed the same rules and the vortex strength and its range were reproduced well. As expected, the flow through the structure, which influenced the flow around the spur dike was noticed.

Vertical, longitudinal cross-sections are presented in Fig. 11 to Fig. 14. Fig. 11 and Fig. 12 show cross-section at $y=0.15 \mathrm{~m}$ for impermeable and permeable structure case respectively. Fig. 13 shows cross-section at $y=0.20 \mathrm{~m}$ for the impermeable case, whilc Fig. 14 shows cross-section at $y=0.20 \mathrm{~m}$ for permeable structure. Easy to recognize is fluctuation of the water surface (especially in the impermeable case, as the presented graphs are plotted for the time $t=8 \mathrm{~s}$ ). At the level $0.1 \mathrm{~m}$ below the surface this fluctuation does not have an effect on vertical velocities. This result is confirmed by the experimental results. Unfortunately limitations of ADV didn't allow to measure velocities higher than $0.1 \mathrm{~m}$ below the surface.

At the upstream-side of the dike in both experiment and simulation - the downward current was seen. It was observed in both impermeable and permeable case, but in the case of solid structure it had slightly larger value. The $z$-direction velocities from simulation and experiment were similar.

Some differences between vertical velocities obtained form experiment and calculations are visible in Figs. 13 and 14. At the upstream side of the dike, in the distance of $5 \mathrm{~cm}$ from the structure (for $y=20 \mathrm{~cm}$ ) for both experimental and numerical results the velocities are almost horizontal. Near the headwall, in the experiment some downward current was recorded close to the riverbed. On the other hand in this area some small upward velocities were simulated.

\section{Conclusions}

The three-dimensional VOF model for free-surface and turbulent flow considering flow in porous media, applied to simulate flow around a spur-dike, gave reasonably good agreement with the experimental results. Although some discrepancy was noted, especially at the downstream-side of the structure, overall image of the flow pattern was reproduced in satisfactory manner. The influence of boundary settings is clearly visible. Although the computational power of modern computers allows performing such a calculation, but still limits the area of calculation and the grid size.

The performed simulation proved good performance of used techniques. Despite of some limitations and discrepancies, the model was already used to simulate the unsteady flow conditions (the surge pass case) and proved its applicability and huge potential in simulating free-surface flows.

With present limitations of the power of computers, future development should include study on the boundary layer application. Influence of the boundaries in the model was significant. Some of the observed discrepancies were caused by the thickness of the cells near the walls treated as the boundary layers. At the same time, effect of numerical smoothing should be eliminated as well. Applicability of presented turbulence model should be verified as well. Some future developments may include testing other turbulence schemes in order to achieve better data. Additional study is necessary to improve presented model in order to avoid those negative features.

As the calculations presented in this paper took enormous time to proceed, future development of the model will include the study on optimization of the method. Speeding up the model is one of the most important goals as the practical application is concerned. With those developments, the model is supposed to be very important for design improvement of hydraulic structures like spurs, bridge abutments, piers and many others.

\section{REFERENCES}

1) Van der Wal M.; Approach to Groyne Innovation in the Netherlands, Workshop proc. of New insights in the physical and ecological processes in groyne fields, Magdeburg, pp. 33-38, 2001. 
2) Lagasse P.F., Zevenbergen L.W., Schall J.D., Clopper P.E. ; Hydraulic Engineering Circular No. 23 (HEC-23); Fed. Hwy. Adm. U.S. Dept. of Transp.; Washington, D.C, 2001.

3) Mioduszewski T., Maeno S., Uema Y.: Influence of the Spur Dike Permeability on Flow and Scouring during a Surge Pass, Proceedings of the International Conference on Estuaries and Coasts, pp.380-388, 2003.

4) Mioduszewski T., Maeno S., Uema Y.: Influence of the Spur Dike permeability on Local Velocities and Scouring; Proceedings of 4th International Symposium on Environmental Hydraulics and 14th Congress of APD, $I A H R$, Vol. 2, pp. 1843-1848, 2004.

5) Nichols B.D., Hirt C.W. and Hotchkiss R.S.: SOLA-VOF: A Solution Algorithm for Transient Fluid Flow with Multiple Free Boundaries, Los Alamos Scientific Laboratory, LA-8355, 1980.

6) Hirt C.W., Nichols B.D.: Volume of Fluid (VOF) Method for the Dynamics of Free Boundaries; Journal of Computational Physics, Vol. 39, pp. 201-225, 1981.
7) Maeno, S., Bierawski, L., Fujita, S.: A Study on Dynamic Behavior of the Seabed around a Seawall under Wave Motion Using the VOF-FEM Model, Proceedings of the 12 th International Offshore and Polar Engineering Conference, Vol.3, pp. 579-603, 2002.

8) Michioku K., Ishigaki, T., Maeno, S., Nanjo, M. and Ikematsu, T.: Experiment and analysis on flow field around and inside a permeable groin, Annual Journal of Hydraulic Engineering, JSCE, Vol.48, pp.pp.799-804, 2004.

9) Chan, E. C., Fue-Sang, Lien and Yovanovich, M.M.: Numerical study of forced flow in a back-step channel through porous layer, Proc. of NTHC'00, 34th ASME National Heat Transfer Conference, pp.1-6, 2000.

10) Hirt C.W.: Heuristic Stability Theory for Finite-Difference Equations, Journal of Computational Physics, Vol. 2, pp. 339-355, 1968.

11) Melville B.W., Coleman S.E.: Bridge Scour; Water Resources Publications, LCC, 2000.

(Received April 15, 2005) 